\title{
Desafíos del profesorado al evaluar competencias en alumnos de bachilleratos profesionales técnicos
}

\author{
Teachers' challenges while assessing \\ professional-technical high school students
}

\author{
CARlos Francisco Lozano Corrales* \\ Rubí Surema Peniche Cetzal*
}

En este documento exponemos los resultados de la investigación cuyo objetivo fue indagar las dificultades que enfrentan los profesores de bachilleratos profesionales técnicos al evaluar competencias de sus estudiantes; para ello, llevamos a cabo entrevistas semiestructuradas con 16 profesores de estos bachilleratos, tanto en planteles cuyos alumnos obtuvieron resultados mayores de la media nacional en exámenes a gran escala como en los cuales los resultados fueron notoriamente más bajos. Tras utilizar el análisis de contenidos en el diseño cualitativo, los hallazgos se representan por medio de seis agrupaciones, correspondientes a las tipificaciones de evaluación diagnóstica, formativa y sumativa, así como autoevaluaciones, coevaluaciones y heteroevaluaciones. Por cada tipo de evaluación presentamos las principales dificultades referidas por los profesores, que van desde la disposición de los estudiantes a ser evaluados hasta la falta de condiciones en los planteles para ejecutar esas evaluaciones. Nuestro estudio corrobora algunos resultados de investigaciones previas y contrasta otros que difieren de lo expuesto por otros autores.

This document shows results from the research objective of inquiring the difficulties expressed by professional-technical high school teachers while assessing their students' competences, both in institutions with good levels measured with worldwide exams and those institutions with low results on them. Through a content analysis in the qualitative design, results are grouped in six assessment types: diagnostic, formative and summative assessment, as well as self, co and hetero assessments. Each of these groups contains teachers most often referred difficulties, from students' unwillingness to be assessed, to institutions' lack of conditions to carry on the assessment. Through the results shown here, some previous research program results can be verified, but also some others are highlighted as they differ, considering the specificity of conditions found in professional-technical high school institutions.

\author{
Palabras clave: \\ evaluación de \\ aprendizajes, \\ educación \\ media superior, \\ enfoque por \\ competencias
}

\section{Keywords: competences assessment, assessment challenges, professional- technical high schools, competences model}

Recibido: 28 de febrero de 2018.| Aceptado para su publicación: 20 de agosto de 2018. DOI: 10.31391/S2007-7033(2018)0051-007

\footnotetext{
* Maestro en Investigación Educativa. Profesor del Departamento de Psicología de la Universidad Autónoma de Aguascalientes. Sus líneas de investigación versan sobre actores educativos, evaluación y bachillerato. Correo electrónico: c.lozano84@hotmail.com/ORCID: https://orcid.org/0000-0002-7159-9001

* Doctora en Educación. Profesora-investigadora en el doctorado en Investigación Educativa, Universidad Autónoma de Aguascalientes. Sus líneas de investigación versan sobre eficacia escolar, bachillerato, liderazgo escolar y evaluación. Correo electrónico: rupeniche81@gmail.com/ORCID: http://orcid.org/0000-0003-0105-6471
} 


\section{INTRODUCCIÓN}

$\mathrm{M}$

éxico ha experimentado importantes cambios económicos y políticos que han repercutido en diferentes esferas sociales, incluyendo la edu-

cativa; en esta una de las decisiones con mayor impacto en el país fue la obligatoriedad de la educación media superior (EMS) a partir de 2012. Según Zorrilla (2010), un elemento notorio en las reformas a los programas educativos del gobierno federal es la presentación e implementación de la Reforma Integral de la Educación Media Superior (RIEMS), inclinada a una formación desde un enfoque basado en competencias, las cuales define como "la integración de habilidades, conocimientos y actitudes en un contexto específico" (SEP, 2008, p. 2). La Subsecretaría de Educación Media Superior (SEMS) (citado en Zorrilla, 2010) lo complementa al señalar que “... las competencias describen lo que se ha estimado necesario con las palabras indispensables; son precisas, directas y concisas y describen únicamente una capacidad y son evaluables" (p. 11). Con esto, se incorporan nuevas maneras de evaluar, lo que, además, encierra un propósito dinámico de formación en competencias y su evaluación.

Lozano (2012) y Silva (2015) afirman que el currículo basado en competencias en el cual se enmarca la RIEMS plantea de modo reduccionista y simplista la búsqueda de compatibilidad escuela-trabajo, además de carecer de bases empíricas. Para Lozano (2012), no será posible su implementación con un marco laboral como el actual, si se considera que el docente no dispone del tiempo que se requiere para incorporarse a capacitaciones y certificaciones que le permitan aplicar de la manera esperable los conocimientos y las habilidades que pudiese obtener en ellas.

Pons y Cabrera (2016) detallan que profesores de EMS de reciente ingreso al campo educativo y hasta los que llevan casi tres décadas en el ejercicio docente han mostrado incertidumbre ante la necesidad de aplicar lo establecido en la RIEMS en su currículo basado en competencias, tomando como referencia los planes y programas que les han sido proporcionados. Estos autores aseguran que es indispensable el estudio del ejercicio docente desde una visión interna de las escuelas, que otorgue cuentas claras de las problemáticas y los retos que estas plantean a los docentes y a la institución.

\section{LA EVALUACIÓN EN EL AULA BASADA EN EL ENFOQUE POR COMPETENCIAS}

Aunque la evaluación en los sistemas educativos está emigrando del énfasis en conocimientos factuales a evaluaciones más integrales de conocimientos y habilidades con la finalidad de superar diversos problemas observados al evaluar, con el enfoque por competencias se han presentado problemas en la forma de evaluar a los estudiantes, como la falta de referencia a los retos del desarrollo personal y la ausencia de metodologías de enseñanza y evaluación pertinentes (Tobón, 2013).

En el contexto mexicano y de manera pragmática, la evaluación del logro de competencias que poseen los alumnos de nivel medio superior muestra problemas para llevarse con fluidez y en forma adecuada. García y Martínez (2014) identificaron que los docentes tienen problemas incluso para definir qué son las competencias, y dieron cuenta que más de $80 \%$ de los docentes entrevistados en su investigación requieren ser capacitados en este tema. 
Esta falta de capacitación ya se preveía como fuente de dificultades en la implementación del modelo por competencias. López y Tinajero (2009) señalan que los problemas que más refieren los profesores para aplicar con efectividad un enfoque educativo basado en competencias tienen que ver con la planeación, información oportuna y capacitación, así como los concernientes a la institución y los alumnos, que incluyen la infraestructura insuficiente, el elevado número de alumnos por grupo, además de la falta de preparación y motivación de los mismos alumnos.

Obaya y Ponce (2010) encuentran que una de las preocupaciones del docente de bachillerato en México es utilizar estrategias de evaluación que le permitan otorgar retroalimentación efectiva al alumno para que logre conexiones entre lo que sabe, lo que entiende, lo que vive y el cómo relacionarlo con los nuevos contenidos en clase. Huerta (2014) expone que el docente usa instrumentos de medición de manera efectiva y se refiere, primordialmente, a exámenes de diversa índole, pruebas objetivas, test instruccionales, inventarios y escalas, que facilitan al docente no solo la representación numeral del nivel de logro del alumno, sino, incluso, sus aptitudes, estilos cognitivos, valores, etcétera.

Villardón (2006) propone centrarse más en el proceso global de la adquisición de competencias y evaluar de manera holística lo que el alumno puede realizar, o no, y considerar siempre la posibilidad de que este pudiera tener las habilidades necesarias, pero no estar acostumbrado a la evaluación específica. Por su parte, para Valverde, Revuelta y Fernández (2012), "la evaluación necesita ser considerada como un proceso de recogida de evidencias [...] y de formulación de valoraciones sobre la medida y la naturaleza del progreso del estudiante, según unos resultados de aprendizaje esperados" (p. 53).

Aunque Valverde, Revuelta y Fernández (2012) describen la evaluación en EMS como posible fuente de motivación para los estudiantes, ya que con cada actividad pueden probar qué han aprendido y cómo podrían realizarlo, existe la posibilidad de que los estudiantes no la signifiquen de esta forma; por ello, puede combinarse con la propuesta de Villardón (2006), quien añade que la evaluación no puede ser fija y predeterminada, sino que el docente debe concebirla de manera más móvil y estar en todo momento listo para recabar datos acerca de los logros en la adquisición de conocimientos, habilidades y actitudes por parte de sus alumnos, siempre enmarcada en lo demostrable en cada momento en el aula y no en una sola exhibición final, por ejemplo, un examen.

La tendencia entre algunos docentes es usar menos exámenes e intentar nuevas técnicas de evaluación. En sincronía con ello, Álvarez (2001) señala que el examen no tiene por qué ser desechado, pues cumple con funciones educativamente válidas, sino que son las maneras en que el profesor aplique los exámenes y los aspectos que con ellos intente medir, así como los usos y abusos de los exámenes, lo que pueden llevarlo a recurrir a estos solo por costumbre de la actividad.

Existe una gran variedad de estrategias de evaluación descritas en detalle en la literatura, que van desde la implementación de proyectos y casos hasta el uso de evaluaciones acordes con el momento en el que se evalúa y el actor a cargo de ella. Según el actor, podemos citar la autoevaluación, coevaluación y heteroevaluación, y de acuerdo con el momento, las evaluaciones diagnóstica, formativa y sumativa. 
La migración de la evaluación convencional que emplea exámenes o rubros como la asistencia y la puntualidad para la ponderación de la calificación ha dado paso a formas más completas y enriquecidas de evaluar, como los casos y proyectos, que pueden ser utilizados en la evaluación siempre y cuando den cuenta fehaciente del constructo o habilidad que se desea evaluar, que se realicen con claridad y permitan tanto al profesor mejorar su propia enseñanza como al alumno hacer conciencia de aquello en lo que puede aún prepararse mejor. Si solo se recurre a ellas con fines de medición, como calificar, cualquier actividad y herramienta de evaluación puede servir para poco o nada en términos educativos (Álvarez, 2001, 2008).

\section{EL ESCENARIO DE ESTUDIO}

En 2012, el examen que corresponde al Programa para la Evaluación Internacional de los Estudiantes (PISA, por sus siglas en inglés) se aplicó a un total de 25,042 estudiantes de EMS en México. Estudiantes de bachilleratos privados destacaron notoriamente por encima de la media nacional; por su parte, los de bachilleratos profesionales técnicos, cuyo subsistema es representado en su mayoría por el Colegio Nacional de Educación Profesional Técnica (Conalep), debido a la cantidad de planteles que existen, obtuvieron bajos resultados.

Aunado a lo anterior, la aplicación de 2015 del Programa Nacional para la Evaluación de los Aprendizajes (PLANEA) muestra que los estudiantes de planteles de Aguascalientes del Conalep lograron resultados más alentadores que los encontrados a nivel nacional en bachilleratos, y mucho mejores si se toman en consideración solo los Conalep, en los que sobresale el incremento del porcentaje de alumnos con resultados en los niveles superiores en matemáticas. En contraste, es notoria la situación opuesta cuando se analizan solo los resultados fuera de la capital del estado, donde la mayoría de los alumnos alcanzaron los primeros dos niveles de logro (INEE, 2016).

Aunque se han publicado diversas críticas sobre los resultados que alcanzaron algunos planteles de formación profesional técnica, de manera específica el Conalep, hay pocos estudios que expliquen las condiciones en las que se encuentran estos establecimientos escolares. De manera general, y considerando diversos subsistemas, Hernández y Andrade (2010) muestran que algunos docentes no presentan una formación pertinente en el enfoque por competencias o no saben cómo aplicarlo en las materias que imparten.

Es importante resaltar que, según la revisión de Hernández y Andrade (2010) respecto al uso de competencias en bachilleratos, el Conalep fue una de las primeras instituciones en implementar la educación basada en competencias, incluso antes de la RIEMS, al buscar establecer un sistema que pudiera formar a sus estudiantes de acuerdo con estándares laborales. Posteriormente, derivado de la citada reforma, el Conalep adoptó el uso de términos y especificaciones que esta contiene.

\section{OBJETIVO DE INVESTIGACIÓN}

Determinar las dificultades que enfrenta el docente al evaluar los aprendizajes en escuelas de nivel medio superior de formación profesional técnica en el enfoque por competencias, según los tipos de evaluación. 


\section{MARCO METODOLÓGICO}

El diseño metodológico del estudio corresponde al enfoque cualitativo, al recabar información en el discurso de los profesores que laboran en contextos y situaciones similares. Con ello, pretendemos reflejar los retos que enfrentan los docentes al evaluar las competencias de sus alumnos de acuerdo con lo establecido en documentos oficiales mexicanos. La intención es que el diseño coincida con la propuesta de Creswell (2002), quien refiere que, para recabar información de este tipo, es preciso "seleccionar las personas o sitios que mejor puedan ayudar a describir nuestro fenómeno [...] para desarrollar un detallado entendimiento que provea información útil, ayude a aprender acerca del fenómeno, y dé voz a personas en silencio" (p. 193).

Cohen, Manion y Morrison (2007) señalan que el enfoque cualitativo parte del supuesto de que el ser humano construye sus propios conocimientos y situaciones y les otorga significado por medio de la propia experiencia; por ello, las realidades son múltiples y guiadas por el contexto donde el individuo se sitúa, y se enriquece al compartir y comparar historias y biografías entre individuos. En este caso, nuestro estudio plasma las experiencias de los docentes al evaluar las competencias adquiridas por sus alumnos durante las sesiones en aula de bachilleratos profesionales técnicos, Conalep.

\section{Participantes}

En el estudio participaron profesores de EMS de formación profesional técnica en el estado de Aguascalientes. Nos centramos en docentes que laboran en el Conalep de Aguascalientes; seleccionamos a ocho profesores de cuatro planteles que se encuentran dentro y ocho profesores de cuatro planteles fuera de la capital del estado. Correspondieron a planteles con altos y bajos puntajes en los resultados de pruebas a gran escala, con la intención de que la selección de los participantes coincidiera con la propuesta de Creswell (2002): el enriquecimiento de la información recabada por medio de la inclusión de experiencias diversas, y que ilustre de manera consistente la situación que se plantea estudiar.

Por cada una de las áreas disciplinarias básicas consideradas en la RIEMS (matemáticas, ciencias experimentales, ciencias sociales y comunicación) del tronco de formación básica del Conalep, solicitamos a los directivos poder entrevistar a un docente por área en cada plantel (ver tabla).

Tabla. Selección de participantes por plantel de estudio y área disciplinar de acuerdo con la RIEMS

\begin{tabular}{|c|c|c|c|c|c|}
\hline Área disciplinar & \multicolumn{5}{|c|}{ Docentes por plantel } \\
\hline & Plantel 1 & Plantel 2 & Plantel 3 & Plantel 4 & Total, área \\
\hline Matemáticas & 1 & 1 & 1 & 1 & 4 \\
\hline Ciencias experimentales & 1 & 1 & 1 & 1 & 4 \\
\hline Ciencias sociales & 1 & 1 & 1 & 1 & 4 \\
\hline Comunicación & 1 & 1 & 1 & 1 & 16 \\
\hline Total por plantel & 4 & 4 & 4 & 4 & \\
\hline
\end{tabular}

Esta selección de participantes nos permitió el análisis de evidencias enfocado a las diferencias entre estrategias utilizadas por los profesores de cada área disciplinaria para evaluar a sus estudiantes a pesar de las dificultades que encuentran. 


\section{Técnicas e instrumentos}

Inicialmente, y de acuerdo con la propuesta de Arthur, Waring, Coe y Hedges (2012), construimos una guía semiestructurada de preguntas abiertas para entrevistar a cada uno de los docentes e indagar con cada participante los temas específicos del interés para nuestro estudio. Brindamos la posibilidad a cada uno de expresarse tan a fondo como considerara prudente, pero también orientando y profundizando en temas a medida que el entrevistador lo creyera pertinente para la recolección de información. Cada entrevista se realizó en una sola sesión por cada participante, acordada según las posibilidades del docente y la conveniencia entre docente e institución; para ello, concertamos las visitas necesarias al plantel según lo requerido por los participantes y el entrevistador.

Finalmente, el procesamiento de la información fue llevado a cabo por medio de la identificación de patrones y temas, además de conjunción, comparación y contraste de las experiencias manifestadas por los docentes.

\section{RESULTADOS}

\section{Dificultades al evaluar, según el tipo de evaluación}

Para abordar los resultados, creímos pertinente retomar la propuesta de tipificación de evaluaciones vertida en la RIEMS (SEP, 2008), que enumera las evaluaciones diagnóstica, formativa y sumativa dado el momento en el cual se lleva a cabo la evaluación, y en relación con el actor que evalúa, considera autoevaluación, coevaluación y heteroevaluación. Es pertinente dada la técnica de análisis de resultados: la comparación y complementación de experiencias de docentes por medio del vaciado de estas en rejilla de doble entrada, que enriquece los grupos de resultados que a continuación compartimos.

\section{Evaluación diagnóstica}

Todos los profesores aseguran que llevan a cabo este tipo de evaluaciones conforme a lo estipulado por la SEP (2011), con el propósito de obtener información sobre el conocimiento de los estudiantes y plantear estrategias de enseñanza según los resultados; sin embargo, existen dos grupos de dificultades que enfrentan al aplicar estas evaluaciones: los alumnos tienen conocimiento de que los resultados bajos en evaluaciones diagnósticas ocasionan también un decremento en el nivel del curso y, de manera intencional, pretenden obtener resultados más bajos: "Sucede muy a menudo, es que hacen referencia, quizá maliciosamente, de que no tienen conocimiento de muchas cosas que quizá tengan para intentar evitar el nivel..." (M1). Con ello, el profesor se ve en la necesidad de diseñar instrumentos de evaluación para alcanzar un diagnóstico fiel y objetivo del nivel de competencias desarrollado por sus estudiantes en forma previa.

En otras ocasiones, el alumno puede evidenciar competencias menos desarrolladas con intención o porque en efecto no recuerda lo que se le pregunta: "Literalmente: ¡tienden a olvidar todo! [...] el hecho de que están en blanco no quiere decir que no sepan: ya cuando uno empieza es cuando ‘ah, sí, ya me acordé!’” (C7); por 
lo tanto, es difícil elegir actividades que demuestren la obtención de competencias previas del joven o confiar en plenitud en estos resultados.

La falta de disposición de los estudiantes para participar en estas evaluaciones, como menciona (C1): "A veces hay muchachos que llegan muy callados, no quieren hablar, no quieren participar [en evaluaciones diagnósticas]..., dificulta al profesor poner en marcha las estrategias que tenía preparadas a manera de diagnóstico, además de que, dado que algunos alumnos son muy tímidos, no se les insiste demasiado para no hacerlos sentir mal. Retomamos lo planteado por la Organización para la Cooperación y el Desarrollo Económicos (OCDE, 2009), y a varios años ya del surgimiento de la RIEMS (SEP, 2008), los profesores continúan reconstruyendo sus prácticas de enseñanza y de evaluación, e intentan que concuerden con el enfoque constructivista de las competencias, además de lograr coherencia entre lo establecido en el modelo educativo y lo que se aplica en el aula.

De acuerdo con los resultados de las evaluaciones diagnósticas y con el desempeño en el transcurso del semestre, muchos estudiantes de primer ingreso no parecen contar con formación previa satisfactoria; se reciben alumnos con conocimientos mucho más bajos de lo esperado, lo que dificulta al profesor enseñar los contenidos de los planes de estudio de las materias del Conalep de la manera en que se estipula. Con ello se ve en la necesidad de incrementar temas a las sesiones y tener que desarrollar y evaluar competencias que deberían haber tenido los alumnos desde antes de ingresar a bachillerato. Así lo mencionan dos participantes:

... hay que trabajar con ellos un poquito bajando del nivel [...] porque sí, de verdad: no sé si sea [...] yo creo por en donde estamos ubicados y el tipo de alumnos que recibimos, ¿verdad? Pero no son alumnos que entiendan (M4).

Nuestro programa se basa en competencias que los alumnos se supone que ya tuvieron en primaria y secundaria; sin embargo, nos encontramos con que estos alumnos no tienen en lo más mínimo las competencias, no sé si es porque no se está trabajando o simplemente los sacan al vapor (C6).

Estas dificultades parecen hacerse más notorias en las materias de español y matemáticas, pues casi todos los profesores de esas asignaturas mencionan que los alumnos de primeros semestres, al ingresar, ignoran contenidos básicos que deberían conocer para llevar las clases de manera fluida. Estos resultados coinciden con el Informe Nacional 2015 del Instituto Nacional para la Evaluación de la Educación (INEE, 2015), dado que una evaluación diagnóstica mal efectuada puede colocar a los alumnos en problemas posteriores para poder seguir el nivel con el que se imparten las clases.

El problema del bajo nivel de desempeño en los alumnos se presenta en planteles que están tanto dentro como fuera de la capital, y se adhiere un problema que refiere un profesor que, aunque labora fuera de la capital, es compartido por los maestros del Conalep en general (M4), situación que pone de manifiesto la dificultad que representa para los docentes el recibir a estudiantes que no están acostumbrados a trabajar en un currículo basado en competencias: es difícil retroceder en temas que debieron conocer los estudiantes desde tiempo antes a la par que avanzan en lo establecido en las secuencias didácticas. Con esto se hace más difícil ajustar las evaluaciones a los tiempos y contenidos planeados, lo que, a su vez, afecta fuertemente los resultados de las evaluaciones de los estudiantes si no se retoman estos temas. 
Álvarez (2008) plantea la dificultad del tránsito a un nuevo modelo y, como observamos en el último comentario referido, a las dificultades del nivel académico con el que ingresan los estudiantes se agrega el referente a cómo los profesores tienen que seguir estrategias de evaluación en el modelo por competencias, aunque no les parezca congruente con la forma en que, a decir de los profesores del Conalep, se llevan los modelos adoptados por las escuelas de las que provienen los estudiantes:

... el contexto en el que nos encontramos [en el municipio] no tienen el hábito de hacer tareas, de entregar trabajos, nada más te guías con una evaluación sumativa, y llegas aquí y obviamente es el lado opuesto [...] desde la secundaria que no tienen una orientación necesaria, entonces, si se cambiara un poquito esa área de la formación desde un inicio, a ir enfocando el alumno tanto en las habilidades, aptitudes y actitudes que tiene, entonces mejoraría todo (M5).

\section{Evaluación formativa}

La referencia más constante en este grupo es la implicación del tiempo que lleva a los docentes la aplicación de la evaluación formativa, dada la gran cantidad de evidencias que deben registrar a diario (C1): “... es cansado, porque tiene uno que estar registrando diario qué se hizo, qué se logró, y ahí vamos identificando si el alumno va avanzando, como te decía, o se estancó, o está retrocediendo". Pons y Cabrera (2016) tienen razón al considerar que aún en la evaluación formativa el profesor se encuentra con problemas al evaluar de acuerdo con lo establecido en la RIEMS, dado que reunir las evidencias de todas las competencias que los profesores evalúan en sus estudiantes requiere demasiado tiempo a los profesores, además de un gran esfuerzo para organizarse.

A lo anterior se suma el factor de grupos numerosos de hasta cincuenta alumnos: es una gran cantidad de evidencias de logro, incluso si únicamente se requiriera una evidencia por cada competencia desarrollada, por medio de cada una de las actividades registradas para evaluación:

... diario tengo que estar evaluando a los muchachos: todos los días se les evalúa en una actividad, en otra actividad, en los procesos que los llevan a lograr un proyecto, o analizar un caso [...] Entonces diario tienes que estar observando, diario hacer anotaciones [...] y sí son evaluaciones continuas [...] obviamente es más trabajo para el maestro [...] yo tengo 210 alumnos. 0 sea, yo tengo que revisar 210 cuentos (M4).

Perrenoud (2006) aborda la dificultad de evidenciar cada competencia en detalle; los maestros consideran que el tiempo es un factor clave con base en el cual tienen que moverse, y les es difícil llevar una evaluación formativa con tantos estudiantes y de la manera en que se considera en el modelo por competencias.

Aunque Abella, Pérez y Hortigüela (2015) parecen acertar al hablar de la objetividad que puede otorgar la evaluación formativa, en algunos casos la opinión de los profesores difiere de estos autores respecto de la disposición de los alumnos a este tipo de evaluaciones, ya que requieren mediar entre las competencias que el estudiante puede evidenciar en una evaluación y las que registra en la plataforma; además, hay que tomar en cuenta que algunos alumnos sí han desarrollado las competencias, pero por alguna razón no las evidencian justo al momento de la evaluación, ya sea por un error ocasionado por un malentendido en la actividad o dar por hecho que ya lo han demostrado antes: "Es muy subjetivo, porque pueden, por ejemplo, 
pasar por un momento crítico, ¡que nos ha pasado a todos! [...] y entonces uno sabe que sí lo saben porque ya lo han hecho, ¡y se los dices... yo se los digo! [...] Es como si en el examen les pasara algo en la cabeza, ¡un candado! Y se bloquean..." (M3).

La investigación de Obaya y Ponce (2010) acerca de las múltiples conveniencias y el gusto de los alumnos por la evaluación formativa reveló que a estos lo que les gusta de este tipo de evaluación es poder cometer errores sin que ello se vea reflejado en calificaciones:

Se hacen como que [gesticulación de duda], y no le entienden mucho, y dicen “ ¿cómo me va a evaluar si nada más le di un resumen?", sí afecta [que no entiendan la evaluación formativa], porque o se quedan atrás, o se quedan rezagados, o no entienden a la hora del proyecto, o cuando les dejas un ensayo individual se quedan así como que [gesticulación de duda], iy te entregan cualquier cosa! Y de todos modos eso lo tienes que evaluar (M8).

Otros profesores comentan que están en desacuerdo en que el reflejo de algunos factores sea nulo en la calificación: “¡No estoy de acuerdo! La educación formativa también tiene que tener el grado de conocimiento [...] o sea, pero también las actitudes, todos los valores y todo [...] Para mí no es correcto [...] tiene que haber un equilibrio [en ponderaciones]" (M3); sin embargo, la actitud que toman los jóvenes con la evaluación formativa, como una falta de compromiso al participar en clase o ante las tareas de evaluación que se les deja, es otro factor notorio en este tipo de evaluaciones, en las cuales no se pueden dejar tareas porque los alumnos no las realizan: “... no los dejo que se lo lleven porque no lo van a hacer, ¡si se los dejo de tarea no lo hacen!" (M3).

En este aspecto es difícil para los profesores evaluar competencias completas solo con el trabajo que los alumnos puedan desarrollar dentro del salón de clase y específicamente en el tiempo de esta, pero, de no hacerlo así, parece que algunos se enfrentan a no recibir los trabajos y la consecuente necesidad de registrarlo de ese modo en las evaluaciones. Esto contrasta con lo expuesto por Valverde, Revuelta y Fernández (2012), quienes plantean la evaluación formativa como fuente de motivación para los estudiantes; sin embargo, no se está dando el caso, ya sea porque no se lleva de manera adecuada o por las mismas aspiraciones de los alumnos, en virtud de que los profesores consideran que muchos de ellos le dan poca importancia a los resultados de las evaluaciones.

Los profesores pueden coincidir con las estrategias de evaluación que plantean las guías, que están basadas en lo que ellos nombran programas de estudio, correspondientes a las secuencias didácticas proporcionadas por el Conalep, y pueden intentar implementarlas del mejor modo; no obstante, en muchas ocasiones no pueden realizarlas tal como se indica (C7). Los profesores se ven en la necesidad de aplicarlas en el momento para poder cumplir con los tiempos obligatorios establecidos en las condiciones que se les señalan; omitir algunas evaluaciones no es la mejor opción, porque después les resulta difícil evidenciar las competencias desarrolladas acumulativamente.

La Dirección General de Bachillerato (SEP, s.f.) ya había dado cuenta de dificultades tanto con los alumnos como con la carencia de infraestructura y mobiliario necesario para las prácticas de enseñanza y evaluación. Los profesores participantes relatan que sus condiciones no les permiten dedicar sesiones plenamente al desarrollo y evaluación 
de prácticas en las que sus alumnos puedan demostrar su nivel de aprendizaje, ya que tienen que resolver esas ausencias durante la clase (C5), lo que resta tiempo para sesiones dedicadas tanto a enseñanza como a evaluación.

\section{Evaluación sumativa}

En el trabajo en el aula, de acuerdo con el modelo por competencias, lo deseable sería que los profesores utilizaran evaluaciones tanto formativas como sumativas; sin embargo, los directivos de los Conalep insisten a los docentes en que eviten las evaluaciones sumativas por medio de pruebas escritas, y que sean sustituidas por estrategias de evaluación más elaboradas, como los proyectos o rúbricas basados en portafolios de evidencias y proyectos individuales y en equipo.

Esta situación puede ser planteada para evitar lo que el Centro Interuniversitario de Desarrollo (CINDA, 2008) describe como un uso desmedido de exámenes y se intenta evitar que los profesores vuelvan a centrarse únicamente en estos instrumentos para evaluar durante el curso. Sin embargo, para algunos profesores esto es una medida impuesta de manera innecesaria por el Colegio, dado que han demostrado que saben evaluar sin exámenes, pero, en ocasiones, los consideran convenientes, aunque no todos los profesores cumplen con esta encomienda (M6).

Evaluar y registrar todos los componentes de las competencias en la evaluación sumativa es difícil para el docente, si tomamos en cuenta que, para ello, se conjuntan algunos temas ya tratados: el tiempo que le requiere, la cantidad de alumnos y la multiplicidad de factores asociados a la calificación, como las condiciones y configuraciones particulares del desempeño de cada uno de los estudiantes (C2).

Respecto al convencimiento de los estudiantes sobre este modelo, trabajar desde este enfoque puede ser poco benéfico para ellos si lo que se pretende es hacerlos responsables, ya que los directores de algunos planteles exigen que se les brinde a los estudiantes la posibilidad de entregar trabajos a destiempo sin que ello tenga consecuencias verdaderas en su formación: “... al muchacho le faltaron cierto trabajo, te lo pueden entregar directamente [...] y tú debes subir la calificación, y yo digo, ¡le dan la oportunidad! En un trabajo no te van a esperar: 'A ver, remédialo y ya te podemos...' (C3).

Este tipo de problemas ya los preveía Villardón (2006) cuando exponía que la evaluación requiere ser dinámica, constante, dado que los profesores se ven en la necesidad de elegir entre beneficiar a los alumnos con evaluaciones que sean sencillas o evaluarlos de manera correcta para colaborar efectivamente en su formación. En este caso, el Conalep es juzgado por la calidad del trabajo de sus egresados (C6).

Los profesores expresan que es difícil registrar los componentes con tres rubros en la plataforma destinada a ello, ya que el rubro menor representa un nivel muy bajo, el siguiente nivel está muy alejado y, a su vez, demasiado cerca del último. Esto lleva al docente a situaciones en las que necesita elegir entre si otorga un insuficiente, que es demasiado bajo para el desarrollo de las competencias del alumno, la excelencia, aun cuando la competencia del alumno no se evidencie así, o la suficiencia, a pesar de que el alumno haya hecho un mejor trabajo, pero sin llegar a la excelencia. El reto principal para los profesores en ese sentido es precisamente lograr el uso deseable de las evaluaciones, es decir, utilizar cualquier estrategia y actividad como una evidencia de evaluación sumativa. 


\section{Autoevaluación}

Aunque las secuencias didácticas proporcionadas por el Conalep y los resultados de la ponderación en sus evaluaciones consideran el uso de la autoevaluación del alumno en varios momentos e, incluso, se le otorga un porcentaje para la calificación numérica generada posteriormente, lo predominante es la creencia de que este tipo de evaluación es demasiado subjetiva (C1). Por ello, los profesores encuentran complicada la promoción de esta evaluación y se les dificulta diseñar estrategias para que los jóvenes se evalúen a sí mismos con objetividad u optan por dejar de considerar los resultados que en estas autoevaluaciones se plasman: aunque la autoevaluación supone una oportunidad para la valoración del alumno acerca de su propio trabajo, a decir de los docentes esto no se refleja en las secuencias didácticas cuando se asigna un porcentaje de calificación numérica a la autoevaluación del estudiante.

Hay un problema más que se presenta debido a la subjetividad de estas evaluaciones, ya que los alumnos más preparados parecen evaluarse a sí mismos mucho más bajos y los menos preparados lo hacen al contrario: "Los que van bajos, jmuy propios, dicen Excelente! [...] y quienes son muy buenos trabajando, siempre excelente, ¡y se ponían suficiente! 0 a veces '90, maestra'" (M6).

Ante estos usos inadecuados de la autoevaluación, a los profesores se les dificulta decidir entre omitir la autoevaluación en sus grupos, utilizarla a pesar de los resultados subjetivos con los que se encuentran, o bien, usarla, pero sin reportar los resultados que los mismos alumnos generan. Valverde, Revuelta y Fernández (2012) exponen que la evaluación puede ser fuente de motivación para el propio alumno. En los hallazgos representados aquí esperábamos corroborar esa afirmación por medio de la autoevaluación, de la que los estudiantes pueden hacer uso como el medio de mejora que proponen estos autores; no obstante, los hallazgos muestran más el deseo del estudiante de beneficiarse que la autocrítica correcta para poder mejorar su desempeño. Así, el alumno desaprovecha la oportunidad de valorar su trabajo y aprender de él.

\section{Coevaluación}

Para este tipo de evaluación existen dos malos usos muy comunes que los estudiantes intentan aplicar en repetidas ocasiones: uno, pretenden verse beneficiados cuando sus compañeros los evalúan, y dos, intentan afectar a los compañeros con los que tienen mala relación. En el primer caso, la referencia es clara acerca de las implicaciones que el uso de la coevaluación tiene, ya que existe "un uso no ético del sistema de evaluación, al alterar ellos mismos los resultados..." (M1), o bien, al expresar comentarios que intentan beneficiar a compañeros con los que se relacionan bien (C2) o afectar a aquellos con los que se llevan mal (C1, M2).

En estas ocasiones es difícil para el profesor encauzar a los alumnos hacia una evaluación más objetiva, apegada a lo que el mismo profesor pretende que se evalúe. Sin importar que los profesores utilicen herramientas como listas de cotejo, parece difícil encontrar las condiciones para que se cumpla la segunda característica que Tobón (2013) propone en la coevaluación, porque los estudiantes no parecen haber desarrollado una conciencia plena de la importancia de sus comentarios. 


\section{Heteroevaluación}

Los profesores saben que la evaluación de sus alumnos por parte de otros profesores es algo deseable: una visión diferente a la del propio docente, enfrascado en sus clases y en lo cotidiano de sus métodos de evaluación, puede otorgar a docente y alumnos la posibilidad de evaluación efectuada desde otro ángulo. Sin embargo, las evaluaciones a cargo de alguien más que pretenden dar evidencia del nivel de logro de los estudiantes no son consideradas viables para los profesores participantes en nuestro estudio, "porque solo ellos conocen el desempeño y el trabajo de cada alumno" (M3).

Por esta razón, los profesores difícilmente piden o aceptan que alguien más lo haga, ya que evalúan aspectos muy específicos de su grupo y en ambientes muy controlados para que no se vea afectada la percepción del desempeño de los alumnos; por ejemplo, en una práctica que se lleva en dos materias en conjunto, cada profesor se enfoca en evaluar solo la parte que le corresponde, o en una actividad, como una feria creada por los estudiantes, se le pide a un profesor que sea juez evaluador, pero únicamente de aspectos estéticos o de organización.

De acuerdo con López y Tinajero (2009), muchos profesores ven la imposición de la RIEMS como un tropiezo que no permite demostrar buenos resultados a los alumnos debido a las discrepancias entre lo establecido en los documentos oficiales y las posibilidades reales en los planteles para llevar a cabo evaluaciones que consideren la transversalidad de los contenidos.

Por otra parte, los participantes en nuestro estudio, en su mayoría, mencionan un problema en común: la falta de costumbre de sus alumnos a contestar pruebas como las de PISA o ENLACE. Por ello, desde hace casi tres años evitan casi por completo que estos se expongan a evaluaciones con características similares y, cuando estas se aplican, los estudiantes muestran incluso desconcierto:

... tanto PLANEA, EXANI, son de evaluación de opción múltiple, y nosotros comúnmente evaluamos a veces con preguntas abiertas, con proyectos [...] realización de esto [...] esos detalles que le dan puntos al alumno; ya cuando viene este tipo de PLANEA, EXANI, jes otro mundo! ¡Es algo completamente distinto a lo que se lleva en toda la carrera! (C7).

Los profesores encuentran difícil otorgarles prácticas como estas a los estudiantes para que estén preparados cuando sean evaluados de esta manera, ya que el mismo Conalep intenta que no se utilice este tipo de herramientas. Aunado a ello, para los profesores estas pruebas revelan deficiencias que deberían tomarse en cuenta, ya que se les considera antipedagógicas e incoherentes con el modelo sobre el cual se intenta formar a los estudiantes:

Pones a los chicos a resolver un examen, por ejemplo, un PLANEA o un EXANI, que, ¡en un Modelo en Competencias no se ve así! [...] trabajando en un Modelo por Competencias que es totalmente diferente, creo yo que habría que tener mucho cuidado, ¿no? [...] y las horas y el tiempo de estar ahí sentados [...] no creo que siquiera eso pedagógicamente esté bien [...] pero los resultados sí luego vienen y [...] pues no suelen salir bien [...] y quieras o no sí nos los desmotiva (M4).

La dificultad para los profesores, en general, es lograr que tanto las evaluaciones efectuadas por ellos mismos como las que llevan a cabo otros compañeros y otras instancias cuenten con lo que el CINDA (2008) propone: hacer uso de métodos de 
evaluación diversos, que sean referenciados a criterios y sean mesurables, sin perder de vista que el trabajo que se lleva dentro del aula puede ser realizado sin tomar como eje principal la evaluación a través de estos métodos.

Respecto a las condiciones en las que laboran, los profesores mencionan tres temas que se reflejan en los resultados de la evaluación de competencias a sus alumnos: la inconformidad por las condiciones económicas en las que laboran; la falta de compromiso de los directivos al no ser partícipes de la formación en el modelo de enseñanza y evaluación por competencias; y la ausencia de oportunidades de formación que les permitan ampliar su conocimiento de estrategias de evaluación pertinentes.

Algunos profesores hablan de cómo inicialmente se veían más involucrados en el desarrollo de instrumentos y estrategias para evaluar a sus alumnos, con la esperanza de que ello colaborara a cambiar sus condiciones laborales, con mejores sueldos y prestaciones, sobre todo con la supuesta homologación de salarios que pretendía la RIEMS, pero ha pasado el tiempo y no ven el resultado de estas promesas (M4).

\section{Diferencias entre planteles}

De manera específica, los argumentos de los profesores de planteles fuera del municipio capital se enfocan en las diferencias de sus estudiantes en las condiciones del contexto social y familiar del que provienen, en las posibles aspiraciones laborales que les refieren los estudiantes, y en las costumbres que tienen los alumnos respecto de su formación en tareas fuera del contexto escolar.

Además del bajo nivel de preparación que los estudiantes muestran al ingresar al Conalep, que ya comentamos en apartados anteriores, para algunos profesores es difícil evaluar a sus estudiantes porque anteponen las condiciones de los jóvenes:

Los chicos de Aguascalientes van a escuelas donde quizás son un poquito más comprometidas, creo yo. ¡Aquí tienen muchos problemas de alcoholismo, hay mucha drogadicción, abuso, abandono...! Entonces, ¡imagínate con la carga que los recibimos! i $i$ aparte los quieres competentes?! ¡¿Cómo les pongo yo una calificación, así, sin importarme?! (M4).

Esta situación y otras similares, expresadas por más de la mitad de los docentes de planteles ajenos a la capital del estado (M1, M4, M5, M7, M8), ocasionan en algunos de los profesores la necesidad de acercarse a los estudiantes, platicar con ellos, y posiblemente beneficiarlos con mejores calificaciones "... porque [...] jal final muchos lo que ocupan nomás es el papel [...] es salir, ya, y poder buscar un trabajo...” (M7).

Finalmente, se encuentra la falta de costumbre de los alumnos para el estudio, lo que algunos profesores consideran una falta de interés en tareas relacionadas con el contexto académico:

Tenemos un problema crítico aquí en el contexto de Conalep porque los alumnos no acostumbran a leer [...] obviamente por el contexto, tiene que ver mucho el contexto en el que estamos [...] hábito en casa no es adquirido [...] tenemos todavía costumbres conservadoras, donde a lo mejor consideramos que la lectura es algo no interesante, no importante, o lo dejamos de lado, entonces traemos ese hábito en casa de no leer, o de pensar que la lectura es aburrida (M5). 
Algunos profesores consideran el poco hábito de la lectura que tienen los estudiantes como un problema muy arraigado que, de tomarse en cuenta en las evaluaciones, pudiera afectar fuertemente a muchos.

\section{CONCLUSIONES}

Las condiciones de las aulas y los laboratorios en los planteles imposibilitan la realización de actividades que ayuden al profesor a evaluar los niveles de adquisición y desarrollo de competencias de sus alumnos; a ello se suma la falta de materiales e instrumentos necesarios para llevar a cabo estas prácticas, los cuales van desde servicios tecnológicos (computadoras, internet, bocinas, proyectores) hasta materiales y herramientas para materias como física, química y mecánica. Estas condiciones son importantes debido a que los profesores tienen la obligación de evaluar competencias en circunstancias específicas que implican el tener cubiertos estos aspectos.

La gran cantidad de alumnos que conforma cada grupo empeora la situación, ya que el docente está obligado a tener evidencia de la evaluación de cada nivel de desarrollo de competencias que registra para cada alumno. Hay un gran número de competencias durante cada curso, y aunque se implementen actividades para evaluar varias de ellas por medio de una sola estrategia, con grupos que tienen más de cincuenta alumnos los profesores requieren grandes cantidades de tiempo para evaluar y registrar los niveles de logro demostrados.

No obstante que la RIEMS (SEP, 2008) plantea una visión del alumno de bachillerato como aquel comprometido con su proceso de aprendizaje, que busca las oportunidades para formarse mejor y dispuesto a los comentarios que se le formulan para mejorar el nivel y la calidad de su educación, en el plano pragmático los profesores de los planteles del Conalep lidian con muchos alumnos que, incluso, limitan las posibilidades de que esto suceda, no solo durante los momentos de enseñanza, sino en las evaluaciones.

Tanto el bajo nivel de conocimiento con el que ingresan algunos estudiantes a los bachilleratos del Conalep como la baja motivación para estudiar que demuestran otros son también elementos que dificultan la práctica de evaluación conforme el proceso educativo avanza. Los profesores que intentan regularizar a estos estudiantes se enfrentan, al poco tiempo, con el requisito de evaluar las competencias que la plataforma exige para su materia, con lo que pudiesen recurrir a evaluaciones ficticias para no verse afectados, o enseñar y evaluar apresuradamente los contenidos, si acaso es posible hacerlo sin la disminución de la calidad. En cambio, si el profesor elige ignorar este bajo nivel de desempeño de los alumnos de nuevo ingreso, pronto se topará con un alto número de estudiantes en riesgo de reprobación y con la necesidad de decidir entre elevar en forma deliberada las calificaciones de sus estudiantes o contribuir a la desmotivación de algunos estudiantes al reprobar su materia.

Finalmente, respecto al tema correspondiente a la formación docente, todos los profesores participantes consideran necesario actualizarse y perciben como una buena oportunidad la que el Conalep les otorga al facilitarles cursos sobre estrategias de enseñanza y evaluación en el enfoque por competencias; sin embargo, sería prudente revisar las posibilidades que estos cursos brindan, ya que estos profesores suelen ser compañeros docentes que dan clases distintas y con 
quienes, en ocasiones, es difícil compartir estrategias que pudieran ser útiles para evaluar en la misma materia. De ser posible, sería pertinente permitir asistir a cursos dedicados más a la formación a profesores que imparten materias similares y que enriquezcan, así, el abanico de estrategias que puedan utilizar para evaluar a los estudiantes del Conalep.

Felipe Martínez Rizo comentó en una entrevista el 6 de noviembre de 2017 para el Consejo Mexicano de Investigación Educativa (COMIE, 2017a) que, de manera general, las evaluaciones no se han utilizado en forma correcta para orientar la toma de decisiones en cuestión de política educativa para impulsar la mejora, a pesar de los esfuerzos honestos que se han hecho al respecto por parte de los organismos correspondientes. Pablo Gentilli también abordó el tema en una ponencia magistral el 21 de noviembre para el mismo Consejo (COMIE, 2017b), y señaló que los resultados de las evaluaciones a gran escala suelen ser tomados por las instituciones como argumento para penalizar a los docentes y como base para tomar decisiones importantes que no son congruentes con los objetivos de esas pruebas.

Destacamos este par de referencias por la relación que guardan con los resultados aquí mostrados, ya que pareciera que los planteles de bachilleratos profesionales técnicos, y en particular sus profesores y estudiantes, han sido castigados con los juicios emitidos en medios masivos de comunicación acerca de los resultados de las evaluaciones a gran escala, o de correspondencias entre estos resultados y la calidad de trabajo que realiza cada uno de los actores en educación en esta institución, sin considerar las especificidades de sus planteles.

\section{REFERENCIAS BIBLIOGRÁFICAS}

Abella, V., Pérez, Á. y Hortigüela, D. (2015). Perspectiva del alumnado sobre la evaluación tradicional y la evaluación formativa. Contraste de grupos en las mismas asignaturas. REICE. Revista Iberoamericana sobre Calidad, Eficacia y Cambio en Educación, vol. 13, pp. 35-48. Recuperado de http://www.redalyc. org/articulo.oa?id=55133776003

Álvarez, J. (2008). Evaluar el aprendizaje en una enseñanza centrada en competencias. En Gimeno (coord.). Educar por competencias, ¿qué hay de nuevo? Madrid: Morata.

Álvarez, J. (2001). Evaluar para conocer, examinar para excluir. Madrid: Ediciones Morata.

Arthur, J., Waring, M., Coe, R. \& Hedges, L. (2012). Research methods and methodologies in education. EUA: SAGE Publications.

CINDA (2008). Diseño curricular basado en competencias y aseguramiento de la calidad en educación. Chile: Fondo de Desarrollo Institucional/MineducChile. Recuperado de https://www.cinda.cl/download/libros/39.pdf

Cohen, L., Manion, L. \& Morrison, K. (2007). Research methods in education (6a. ed). EUA: Routledge.

COMIE, AC (productor) (2017, 21 de noviembre). La universidad frente a los desafíos sociales en México. Video podcast. Recuperado de https://www.youtube.com/watch?v=V1sBnd4zsrc 
COMIE,AC (productor) (2017,6 de noviembre) Entrevista a Felipe Martínez Rizo. Video podcast. Recuperado de https://www.youtube.com/watch?v=7DjIeS1jz_4

Creswell, J. (2002). Educational research. Planning, conducting, and evaluating quantitative and qualitative research. EUA: Pearson Education.

García, A. y Martínez, L. (2014). El constructo “competencias” en docentes de bachillerato. Entreciencias: Diálogos en la Sociedad del Conocimiento, núm. 2, pp. 163-170. Recuperado de http://www.redalyc.org/articulo. oa?id=457645126004

Hernández, S. y Andrade, R. (2010). El enfoque de competencias y el currículum del bachillerato en México. Revista Latinoamericana de Ciencias Sociales, $\mathrm{Ni}$ ñez y Juventud, núm. 8, pp. 481-508. Recuperado de http://www.redalyc.org/ articulo.oa?id=77315079023

Huerta, L. (2014). La enseñanza, el aprendizaje y la evaluación de competencias en el aula. En Jaik y Málaga (coords.). Las competencias y su relación con... La gestión, la investigación, la docencia, el desarrollo profesional. México: REDIE.

INEE (2016). Base de datos de resultados de examen PLANEA 2015. México. Recuperado de http://www.inee.edu.mx/index.php/planea/bases-de-datosplanea

López, G. y Tinajero, G. (2009). Los docentes ante la reforma del bachillerato. Revista Mexicana de Investigación Educativa, vol. 14, pp. 1191-1218. Recuperado de http://www.redalyc.org/articulo.oa?id=14011808009

Lozano, A. (2012). Límites de la reforma en educación media superior (entrevista). Perfiles Educativos, vol. 34, pp. 164-169.

Obaya, A. y Ponce, R. (2010) Evaluación del aprendizaje basado en el desarrollo de competencias. ContactoS, vol. 76, pp. 31-37.

OCDE (2009). Los docentes son importantes. Atraer, formar y conservar a los docentes eficientes. Recuperado de https://www.google.com.mx/url?sa= $\mathrm{t} \& \mathrm{rct}=\mathrm{j} \& \mathrm{q}=\& \mathrm{esrc}=\mathrm{s} \&$ source $=$ web $\& \mathrm{~cd}=1 \& \mathrm{cad}=\mathrm{rja} \& \mathrm{uact}=8 \& \mathrm{ved}=0 \mathrm{ahUK}$ Ewjnqaiy2 efPAhWCJcAKHeaZDrUQFggdMAA\&url=http \%3A\%2F\%2Fw ww.waece.org\%2Fenciclopedia\%2F $\% 2$ FLos $\% 2520$ docentes $\% 2520$ so n\%2520importantes.pdf\&usg=AFQjCNE9h2SQ5te6m7aDG-XcLCsQ1eSCw\&sig2=j889QMEWynzHxqkZE9KQCQ

Perrenoud, P. (2006). Construir competencias desde la escuela. Santiago de Chile: Noreste.

Pons, L. y Cabrera, J. (2016). La formación docente ante una reforma educativa: estudio en escuelas de nivel medio superior en Chiapas, México. Olhar de Professor, pp. 251-276.

SEP (2011). Lineamientos de evaluación del aprendizaje. México: Dirección General de Bachillerato, SEP.

SEP (2008, 26 de septiembre). Acuerdo número 442 por el que se establece el Sistema Nacional de Bachillerato en un marco de diversidad. Diario Oficial de la Federación. Poder Ejecutivo.

SEP (s.f.). Lineamientos para la práctica educativa a partir del enfoque educativo basado en competencias. Coloquio de Experiencias Docentes. México.

Silva, C. (2015). La Reforma Curricular en Competencias: la experiencia en las preparatorias federales por cooperación. Education Policy Analysis Archives/ Archivos Analíticos de Políticas Educativas, pp. 1-29. Recuperado de http:// www.redalyc.org/articulo.oa?id=275041389038 
Tobón, S. (2013). Formación integral de competencias. Pensamiento complejo, currículo, didáctica y evaluación (4⿳亠丷a . ed). Bogotá, Colombia: Ecoe.

Valverde, J., Revuelta, F. y Fernández, M. (2012). Modelos de evaluación por competencias a través de un sistema de gestión de aprendizaje. Experiencias en la formación inicial del profesorado. Recuperado de http://dialnet.unirioja.es/ servlet/articulo?codigo $=4772438$

Villardón, L (2006). Evaluación del aprendizaje para promover el desarrollo de competencias. Educatio Siglo XXI, núm. 24, pp. 57-76.

Zorrilla, J. F. (2010). El futuro del bachillerato mexicano y el trabajo colegiado. Lecciones de una intervención exitosa. México: ANUIES. 